International Mathematical Forum, 2, 2007, no. 47, 2339 - 2346

\title{
A Numerical Solution of the One-Dimensional Inverse Parabolic Problem Using Chebyshev T Polynomials
}

\author{
H. Molhem \\ Department of Physics \\ Slamic Azad University of Karaj Branch, Iran \\ molhem@kiau.ac.ir \\ R. Pourgholi \\ Department of Physics \\ Slamic Azad University of Karaj Branch, Iran \\ pourgholi@iust.ac.ir \\ D. Momeni \\ Department of Physics \\ Slamic Azad University of Karaj Branch, Iran \\ davood_momeni@yahoo.com
}

\begin{abstract}
In this paper, a numerical solution of an inverse non-dimensional heat conduction problem will be considered. By using a sensor located at a point inside the body and measuring the temperature at a point $x=x_{1}, 0<x_{1}<1$, and applying the Chebyshev polynomials base function to the inverse heat conduction problem, we determine a stable numerical solution to this problem.
\end{abstract}

Mathematics Subject Classification: 35R30

Keywords: Inverse heat conduction problem, Existence, Stability 


\section{Introduction}

The numerical solution of the inverse heat conduction problem (IHCP) requires to determine an unknown boundary condition from an additional information. Inverse heat conduction problems have many applications in various branches of science and engineering. Mechanical and chemical engineers, mathematicians and specialists in many other sciences branches are interested in inverse problems, each with different application in mind [1]-[6].

In this work, by using the ChebyshevT-set a stable solution for an inverse heat conduction problem is presented.

\section{Problem statement}

In this section, let us consider the following IHCP

$$
\begin{aligned}
u_{t} & =u_{x x}, & & 0<x<1, \quad 0<t<T_{0}, \\
u(x, 0) & =f(x), & & 0<x_{1}<x<1, \\
u\left(x_{1}, t\right) & =g(t), & & 0<t<T_{0}, \\
u_{x}(1, t) & =h(t), & & 0<t<T_{0},
\end{aligned}
$$

where $T_{0}$ is a given positive constant, and the sensor is located at $x_{1}, 0<$ $x_{1}<1$, for measuring temperature, at this point, $f(x)$ is piecewise-continuous known function, $g(t)$ and $h(t)$ are infinitely differentiable functions, while the temperature $u(x, t)$ and heat flux $u_{x}(0, t)$ are unknown which remain to be determined. The problem (1)-(4) may be divided into two separate problems. The first problem is

$$
\begin{aligned}
u_{t} & =u_{x x}, & & x_{1}<x<1, \quad 0<t<T_{0}, \\
u(x, 0) & =f(x), & & x_{1}<x<1, \\
u\left(x_{1}, t\right) & =g(t), & & 0<t<T_{0}, \\
u_{x}(1, t) & =h(t), & & 0<t<T_{0} .
\end{aligned}
$$

This problem may be analyzed as a direct problem, for the portion of the body from $x=x_{1}$ to $x=1$ with known boundary conditions. There is a unique stable solution to the direct problem (5)-(8)[5]. The second problem is the 
following IHCP

$$
\begin{aligned}
u_{t} & =u_{x x}, & 0<x<x_{1}, & 0<t<T_{0}, \\
u\left(x_{1}, t\right) & =g(t), & 0<t<T_{0} . &
\end{aligned}
$$

In the next section, the above IHCP will be considered, the heat in the body and heat flux at the boundary $x=0$ will be obtained by solving this problem numerically.

\section{Theorem 2.1 (Existence)}

For piecewise-continuous functions $f(x), g(t)$, and $h(t)$ on their domains, the problem (5)-(8) has a unique solution [7].

\section{Numerical solution of the IHCP (9)-(10)}

In order to solve the problem (9)-(10) we need an extra condition, and we choose the condition

$$
u_{x}\left(x_{1}, t\right)=k(t), \quad 0 \leq t \leq T_{0},
$$

where $k(t)$ can be obtained from the solution of the direct problem (5)-(8). The problem (9)-(11) is a Cauchy problem. The solution of this problem, exists and is unique but not always stable [7]. In this work, stable solution will be given to the Cauchy problem (9)-(11). Now using the transformations

$$
\tau=-1+\frac{2}{T_{0}} t
$$

and

$$
T(x, \tau)=u\left(x, \frac{(1+\tau) T_{0}}{2}\right)
$$

(9)-(11) becomes

$$
\begin{aligned}
T_{\tau} & =\frac{T_{0}}{2} T_{x x}, \quad 0<x<x_{1}, \quad-1<\tau<1, \\
T\left(x_{1}, \tau\right) & =G(\tau), \quad-1 \leq \tau \leq 1, \\
T_{x}\left(x_{1}, \tau\right) & =K(\tau), \quad-1 \leq \tau \leq 1 .
\end{aligned}
$$


This problem is called a Cauchy problem. Now, we seek a solution for this problem in the form

$$
T(x, \tau)=\sum_{i=0}^{\infty} \sum_{j=0}^{\infty} C_{i j} T_{j}(\tau)\left(x-x_{1}\right)^{i},
$$

where $T_{j}(\tau)$ is a ChebyshevT polynomials of degree $j$, and $C_{i, j}$ are constant numbers which must be determined. By putting (17) in (14) and equating the coefficient of each power of $\left(x-x_{1}\right)^{i}$ to zero, we find

$$
C_{i+2, j}=\frac{2 T_{j+1}^{\prime}(\tau)}{T_{0}(i+1)(i+2) T_{j}(\tau)} C_{i, j+1} .
$$

From

$$
T\left(x_{1}, \tau\right)=\sum_{j=0}^{\infty} C_{0, j} T_{j}(\tau)=G(\tau)
$$

we conclude that

$$
C_{0,0}=\frac{1}{\pi} \int_{-1}^{1} \frac{G(\tau)}{\sqrt{1-\tau^{2}}} d \tau
$$

and

$$
C_{0, j}=\frac{2}{\pi} \int_{-1}^{1} \frac{G(\tau) T_{j}(\tau)}{\sqrt{1-\tau^{2}}} d \tau .
$$

Similarly from $T_{x}\left(x_{1}, \tau\right)=K(\tau)$ we obtain

$$
C_{1,0}=\frac{1}{\pi} \int_{-1}^{1} \frac{K(\tau)}{\sqrt{1-\tau^{2}}} d \tau,
$$

and

$$
C_{1, j}=\frac{2}{\pi} \int_{-1}^{1} \frac{K(\tau) T_{j}(\tau)}{\sqrt{1-\tau^{2}}} d \tau .
$$

Substituting the above results in (17), we find

$$
\begin{gathered}
T(x, \tau)=\sum_{s=0}^{\infty} \sum_{j=0}^{\infty}\left(\frac{2}{T_{0}}\right)^{s}\left(\prod_{k=1}^{s} \frac{T_{j+k}^{\prime}(\tau)}{T_{j+k-1}(\tau)}\right) \\
\left(\frac{C_{0, j+s}}{(2 s) !} T_{j}(\tau)\left(x-x_{1}\right)^{2 s}+\frac{C_{1, j+s}}{(2 s+1) !} T_{j}(\tau)\left(x-x_{1}\right)^{2 s+1}\right)
\end{gathered}
$$

Now, if $G(\tau)$ and $K(\tau)$ belong to Holmgren class $H\left(x_{1}, 1, C_{1}, 0\right)$ [5] then $(23)$ is a solution to the problem (14)-(16) [5]. Therefore, if we approximate $G(\tau)$, 
$K(\tau)$ by polynomials of degree $n$, then by the orthogonality of ChebyshevT polynomials with respect to the weight function $\left(1-\tau^{2}\right)^{-1 / 2}$, and the following properties

$$
\int_{-1}^{1} \frac{G(\tau) T_{j+s}(\tau)}{\sqrt{1-\tau^{2}}} d \tau=0, \quad j+s>n
$$

and

$$
\int_{-1}^{1} \frac{K(\tau) T_{j+s}(\tau)}{\sqrt{1-\tau^{2}}} d \tau=0, \quad j+s>n
$$

we will obtain an approximate solution in the form

$$
\begin{gathered}
T(x, \tau) \cong \sum_{s=0}^{n} \sum_{j=0}^{n}\left(\frac{2}{T_{0}}\right)^{s}\left(\prod_{k=1}^{s} \frac{T_{j+k}^{\prime}(\tau)}{T_{j+k-1}(\tau)}\right) \\
\left(\frac{C_{0, j+s}}{(2 s) !} T_{j}(\tau)\left(x-x_{1}\right)^{2 s}+\frac{C_{1, j+s}}{(2 s+1) !} T_{j}(\tau)\left(x-x_{1}\right)^{2 s+1}\right)
\end{gathered}
$$

for the problem (14)-(16). The unknown heat flux at $x=0$ will be obtained by differentiation of (23) with respect to $x$ and putting $x=0$.

Now, by giving the following theorem the stability of solution (23) will be proved.

Theorem 3.1 If $G(\tau)$ and $K(\tau)$, belong to Holmgren class $H\left(x_{1}, 1, C_{1}, 0\right)$ and $G(\tau)$ and $K(\tau)$, are approximated by two polynomials of degree $n$, then the approximate solution (23) is stable.

Proof. Let us consider $T$ as a solution in the form (23) and $T^{*}$ as a perturb solution of IHCP (9)-(11). Then in order to prove the stability of solution (23), we have to show that for any given $\alpha$ if

$$
\begin{gathered}
\left|G(\tau)-G^{*}(\tau)\right|<\alpha, \\
\left|K(\tau)-K^{*}(\tau)\right|<\alpha,
\end{gathered}
$$

then there exist positive numbers $\beta_{1}$ and $\beta_{2}$ such that

$$
\begin{gathered}
\left|T(0, \tau)-T^{*}(0, \tau)\right|<\beta_{1}, \\
\left|T_{x}(0, \tau)-T_{x}^{*}(0, \tau)\right|<\beta_{2} .
\end{gathered}
$$

Using the equation (23), we obtain 


$$
\begin{gathered}
\left|T(0, \tau)-T^{*}(0, \tau)\right| \leq \sum_{s=0}^{\infty} \sum_{s=0}^{\infty}\left|T_{j}(\tau)\right|\left(\frac{2}{T_{0}}\right)^{s}\left|\prod_{k=1}^{s} \frac{T_{j+k}^{\prime}(\tau)}{T_{j+k-1}(\tau)}\right| \\
{\left[\frac{1}{(2 s) !} \int_{-1}^{1} \frac{\left|G(\tau)-G^{*}(\tau)\right|\left|T_{j+s}(\tau)\right|}{\sqrt{1-\tau^{2}}} d \tau+\frac{1}{(2 s+1) !}\right.} \\
\left.\int_{-1}^{1} \frac{\left|K(\tau)-K^{*}(\tau)\right|\left|T_{j+s}(\tau)\right|}{\sqrt{1-\tau^{2}}} d \tau\right]
\end{gathered}
$$

from which, we find

$$
\left|T(0, \tau)-T^{*}(0, \tau)\right| \leq \sum_{s=0}^{n} \sum_{j=0}^{n}\left(\frac{3}{\pi}\right)\left(\frac{2}{T_{0}}\right)^{s} M M_{2}\left(\frac{\alpha}{(2 s) !}+\frac{\alpha}{(2 s+1) !}\right),
$$

where

$$
\left|\int_{-1}^{1} \frac{1}{\sqrt{1-\tau^{2}}} d \tau\right|=M_{2}
$$

Finally

$$
\left|T(0, \tau)-T^{*}(0, \tau)\right| \leq \alpha M_{3}
$$

This completes the proof of stability of solution(23).

\section{A test problem}

the purpose of this section is to illustrate the applicability of the method. This may be done by given some examples.

Example 4.1 In this example we solve the problem (9)-(11) with given data

$$
\begin{gathered}
u\left(x_{1}, t\right)=x_{1}^{2}+2 t, \quad 0 \leq t \leq T_{0}, \\
u_{x}\left(x_{1}, t\right)=2 x_{1}, \quad 0 \leq t \leq T_{0} .
\end{gathered}
$$

Table 1. The Temperature and heat flux on the boundary $x=0$ for $T_{0}=2$ when $x_{1}=.25$.

\begin{tabular}{|c|cc|cc|}
\hline & Numerical solution & \multicolumn{2}{|c|}{ Exact solution } \\
$t$ & $u(0, t)$ & $u_{x}(0, t)$ & $u(0, t)$ & $u_{x}(0, t)$ \\
\hline 0.025 & 0.05 & 0.0 & 0.05 & 0.0 \\
0.225 & 0.45 & 0.0 & 0.45 & 0.0 \\
0.425 & 0.85 & 0.0 & 0.85 & 0.0 \\
0.625 & 1.25 & 0.0 & 1.25 & 0.0 \\
0.825 & 1.65 & 0.0 & 1.65 & 0.0 \\
1.025 & 2.05 & 0.0 & 2.05 & 0.0 \\
\hline
\end{tabular}


Example 4.2 In this example, we consider the problem (9)-(11) with given data

$$
\begin{array}{cc}
u\left(x_{1}, t\right)=\exp (-t) \sin x_{1}, & 0 \leq t \leq T_{0}, \\
u_{x}\left(x_{1}, t\right)=\exp (-t) \cos x_{1}, & 0 \leq t \leq T_{0} .
\end{array}
$$

Table 2. The Temperature and heat flux on the boundary $x=0$ for $T_{0}=2$ when $x_{1}=.25$.

\begin{tabular}{|c|cc|cc|}
\hline & Numerical solution & & Exact solution & \\
$t$ & $u(0, t)$ & $u_{x}(0, t)$ & $u(0, t)$ & $u_{x}(0, t)$ \\
\hline 0.025 & $1.40 \times 10^{-5}$ & 0.975091 & 0.0 & 0.97531 \\
0.225 & $1.19 \times 10^{-5}$ & 0.798218 & 0.0 & 0.798516 \\
0.425 & $1.14 \times 10^{-5}$ & 0.653941 & 0.0 & 0.65377 \\
0.625 & $1.77 \times 10^{-6}$ & 0.535288 & 0.0 & 0.535261 \\
0.825 & $1.15 \times 10^{-6}$ & 0.438252 & 0.0 & 0.438235 \\
1.025 & $1.64 \times 10^{-7}$ & 0.358794 & 0.0 & 0.358796 \\
\hline
\end{tabular}

\section{References}

[1] J. R. Cannon, The One-Dimensional Heat Equation, Addison Wesley, Reading, MA, 1984.

[2] A. Shidfar and G.R. Karamali , Numerical solution of inverse heat conduction problem with nonstationary measurements, Applied Mathematics and Computation, Volume 168, Issue 1, 1 September 2005, Pages 540-548.

[3] J. V. Beck, B. Blackwell, Ch. R. St. Clair Jr., Inverse Heat Conduction John Wiley and Sons, Inc. 1985.

[4] Abdollah Shidfar and Ali Zakeri, A numerical technique for backward inverse heat conduction problems in one-dimensional space, Applied Mathematics and Computation, Volume 171, Issue 2, 15 December 2005, Pages 1016-1024.

[5] A. Shidfar, R. Pourgholi and M. Ebrahimi , A Numerical Method for Solving of a Nonlinear Inverse Diffusion Problem, Computers and Mathematics with Applications, Volume 52, Issues 6-7, September-October 2006, Pages 1021-1030 . 
[6] Friedman A., Partial Differential Equations of Parabolic Type. PrenticeHall, Englewood Cliffs, NJ (1964).

[7] A. N. Tikhonov, V. Y. Arsenin, Solutions of Ill-Posed Problems, V. H. Winston and Sons, 1977.

[8] A. Shidfar and R. Pourgholi, Numerical approximation of solution of an inverse heat conduction problem based on Legendre polynomials, Applied Mathematics and Computation, Volume 175, Issue 2, 15 April 2006, Pages 1366-1374.

Received: February 19, 2007 